\title{
Robot-Assisted Salvage Prostatectomy: Evaluation of Initial Patient-Reported Outcomes
}

Seth A. Strope, M.D., M.P.H., Michael Coelho, B.A., David P. Wood, M.D., and Brent K. Hollenbeck, M.D., M.S.

\begin{abstract}
Background and Purpose: For patients who experience a localized recurrence after definitive radiation therapy for prostate cancer, salvage prostatectomy provides a chance for cure. We sought to assess whether robot assistance would decrease the technical challenges and mitigate the considerable morbidity associated with the procedure.

Patients and Methods: Using institutional data, we identified six patients who underwent robot-assisted prostatectomy after definitive radiation therapy. For all patients, preoperative and postoperative quality of life were measured using the Sexual Health Inventory for Men and the Expanded Prostate Cancer Index Composite (EPIC). Further, intraoperative and postoperative complications were assessed.

Results: Functional status of patients before robot-assisted salvage prostatectomy is compromised. Three of the six patients had extremely poor sexual function before surgery (EPIC sexual domain $<50$ ), and three-quarters had significant irritative symptoms (mean EPIC urinary irritation score 60.5). Surgery was performed safely with no intraoperative complications. Postoperative complications developed in three patients, all of whom were managed conservatively. Of the six patients, four (75\%) remain free of disease; however, incontinence and erectile dysfunction were evident in all, to some degree.

Conclusions: Salvage robot-assisted radical prostatectomy is a safe and effective modality for salvaging patients with localized prostate cancer after radiation. Morbidity remains high, however, likely secondary to the consequences of radiation.
\end{abstract}

\section{Introduction}

A LTHOUGH RADIATION can achieve prostate cancer control in some patients, $10 \%$ to $30 \%$ of patients have biochemical recurrence and may need further therapy. ${ }^{1,2}$ Current systemic therapy, hormonal or chemotherapeutic, provides palliation but not cure. Thus, for patients with localized disease recurrence and a relatively long life expectancy, surgical removal of the prostate offers time-tested curative therapy in selected patients. ${ }^{3}$

Despite the success of salvage prostatectomy in cancer control, few patients opt for this therapy. Complications after surgery may dissuade some patients from embracing treatment. In early series, salvage surgery frequently resulted in incontinence and erectile dysfunction. ${ }^{4}$ Intraoperative complications were also frequent, with $33 \%$ of patients experiencing a major complication, and up to $10 \%$ having a rectal injury. ${ }^{5}$ While these operative complications have decreased in more recent reports, erectile dysfunction and incontinence continue to be major concerns. ${ }^{6}$
Recently, robot-assisted prostatectomy has emerged as an alternative to open radical prostatectomy for these patients. Case reports and small series have addressed the feasibility of the robot-assisted approach in patients in whom radiation therapy had failed. ${ }^{7,8}$ With greater visualization afforded through the robotic approach, intraoperative and postoperative complications may be minimized. To better understand the safety and effectiveness of robot-assisted salvage prostatectomy, we reviewed our results with our first six patients who were treated with this approach using validated measures of quality of life.

\section{Patients and Methods}

We queried our institutional prostate cancer database for patients who underwent a radical prostatectomy after radiation therapy. Operative, pathologic, and quality-of-life data are collected in a prospective and updated manner in this database. Quality-of-life assessments were performed by the patient using the validated Expanded Prostate Index

Department of Urology, University of Michigan Health System, Ann Arbor, Michigan. 
Composite (EPIC) ${ }^{9}$ and Sexual Health Inventory for Men (SHIM) instruments. ${ }^{10}$ Patients completed the self-administered questionnaires preoperatively, and at 3 and 9 months postoperatively.

Patients were considered for salvage prostatectomy if they had biopsy documented locally recurrent or radiation resistant prostate cancer. Only patients with no evidence of metastatic disease on bone scan and CT scan were candidates for salvage prostatectomy. Five patients underwent standard extraperitoneal robot-assisted radical prostatectomy as described previously, ${ }^{11}$ and a single patient had an intraperitoneal approach. This study was completed as part of an Institutional Review Board-approved study on prostate cancer quality of care at the University of Michigan.

\section{Results}

The mean preoperative prostate-specific antigen (PSA) level was $9.3 \mathrm{ng} / \mathrm{mL}$ (range 2.9-30 mL). Two patients had been treated with brachytherapy, and four had received external beam therapy. The original biopsy grade was 6 in three patients, and 7 in three patients. Three of the six patients had a postradiation biopsy where there was no significant radiation artifact. In these patients, the postradiation clinical grades were 7,8 , and 9 .

Surgical technique and outcomes were consistent across the series. The mean operative time was 356 minutes (range 304$430 \mathrm{~min}$ ). No rectal injuries or other intraoperative complications were experienced. Mean estimated blood loss was $280 \mathrm{~mL}$ (range 50-800 mL). No patients received blood transfusions. Patients stayed a mean of 2 days in the hospital (range 1-2 d). One patient had a partial nerve-sparing procedure, and the rest were non-nerve-sparing. Two patients experienced transient urine leaks after the surgery that resolved by postoperative day 2 with conservative management. Late complications were experienced by two of the six patients, with the patients experiencing a bladder neck contracture and posterior urethral distraction, respectively.

Preoperative urinary and sexual function were compromised in all patients. Most patients had some degree of erectile dysfunction, with a mean SHIM of 17.8 (range 11-25). The EPIC sexual function domain echoed these findings with a mean score of 70 (range 0-88). Urinary function as measured by EPIC also revealed significant dysfunction. This dysfunction mainly related to increased urgency and frequency of urination (mean irritation score 60.5; range 0-100).

Postoperative urinary and sexual function continued to be compromised (Fig. 1). At the 3-month follow-up visit, all patients were incontinent. All patients were using three pads per day. By 1 year, this had improved to 2.3 pads per day in the four patients available for evaluation. Two patients underwent male sling procedures after 1 year of follow-up for their incontinence with resolution of symptoms. In three patients with completed SHIM questionnaires at 1 year, all reported erectile dysfunction (SHIM range 1-20).

The final pathology report revealed organ-confined disease in five patients and seminal vesicle invasion in one patient. Margins were negative in all but one patient, who had a focal positive margin. No patient had lymph node involvement. Biochemical disease recurrence occurred in two patients in whom a detectable PSA level was found at the initial 6-week

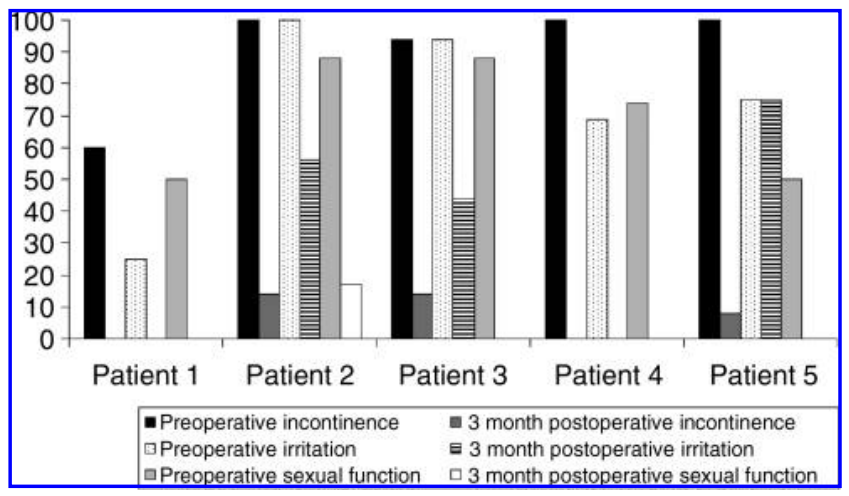

FIG. 1. Preoperative and 3-month postoperative urinary and sexual function. Most patients had compromised urinary and sexual function before their robot-assisted salvage prostatectomy. The preoperative urinary symptoms were irritative. Patients also had compromised sexual function, with only three patients having sexual domain scores greater than 50. Patient 6 had very poor preoperative and postoperative functioning, and full domain scores could not be calculated.

postoperative check. All other patients remain without evidence of disease recurrence at a mean follow-up of 15 months.

\section{Discussion}

Salvage robot-assisted radical prostatectomy appears to be associated with high rates of urinary and sexual dysfunction, which suggests that its advantages of visualization may not translate into better functional outcomes in this population. The advantages of robotics, however, may minimize the risk of rectal injury, perhaps the most catastrophic complication of the surgery. Of note, many of these patients had significant preexisting urinary and sexual dysfunction, likely related to previous therapy. In this select population, however, good cancer control can be achieved with the technique, and functional outcomes improve with time. Further, these patients do respond well to secondary interventions for urinary incontinence and sexual dysfunction.

Functional outcomes after salvage radical prostatectomy have been much worse than for primary therapy. ${ }^{4-6}$ Even the most contemporary series show incontinence in $61 \%$ and erectile dysfunction in $72 \%$ of patients. ${ }^{6}$ Such poor results have prompted investigation into use of robot assistance to improve outcomes. ${ }^{7}$ Yet, the lack of tactile feedback and the fibrosis resulting from radiation therapy may make the procedure as technically demanding as the open procedure. To date, only one series has been published for salvage prostatectomy with robot assistance. ${ }^{8}$ Similar to our results, patients experienced problems with early incontinence and sexual dysfunction. Through our use of validated patient-reported outcome instruments, we establish that many of these urinary and sexual difficulties are direct results of already impaired preoperative function. Regardless of technique, preexisting functional deficits will diminish the efficacy of salvage surgical therapy.

With many patients experiencing poor outcomes after salvage prostatectomy, salvage cryotherapy has drawn increased attention. Using cryotherapy, fewer than 5\% of patients experience urinary incontinence ${ }^{12-14}$; however, rectal 
fistulas (in approximately 1\%) and sexual dysfunction continued to occur. ${ }^{12-14} \mathrm{~A}$ larger concern with cryotherapy is that between $14 \%$ and $33 \%$ of patients will have a positive biopsy from the prostate after salvage therapy. ${ }^{12,15}$ One proposed reason for the low efficacy of the therapy is that prostate cancer is often found close to the urethra. ${ }^{16}$ Thus, efforts to spare the urethra through urethral warmers in cryotherapy may impair cancer control. Salvage radical prostatectomy avoids these potential limitations but increases the risk of urinary incontinence compared with salvage cryotherapy.

Current treatment of patients with local therapy after definitive prostate radiation therapy is suboptimal. Trade-offs between quality of life and effectiveness of therapy must be made. Further, even when carefully selected, many men who undergo salvage therapy ultimately manifest persistent disease. We found that there was biochemical recurrence after surgery in one-third of our patients. These results mirror those of both robot-assisted $(30 \%)^{8}$ and open series $(46 \%) .{ }^{17}$ Current staging modalities do not allow good stratification of patients into those who would respond to salvage therapy and those who would not. To improve the outcomes of salvage therapy, more rapid assessment of radiation failure and continued refinement in surgical technique are needed.

\section{Conclusions}

Salvage robot-assisted radical prostatectomy represents an effective modality for the treatment of patients with localized prostate cancer after definitive radiation therapy. Complication rates with the procedure are low; however, both urinary and sexual dysfunction occur commonly. Many patients have preexisting urinary and sexual dysfunction as a result of radiation therapy, and this dysfunction adversely affects the postsalvage therapy quality of life. Continued refinement of both patient selection and surgical technique is necessary to improve the functional and oncologic outcomes of salvage therapy.

\section{Disclosure Statement}

No competing financial interests exist.

\section{References}

1. Ragde H, Korb LJ, Elgamal AA, et al. Modern prostate brachytherapy. Prostate specific antigen results in 219 patients with up to 12 years of observed follow-up. Cancer 2000;89:135-141.

2. Kuban DA, Thames HD, Levy LB, et al. Long-term multiinstitutional analysis of stage T1-T2 prostate cancer treated with radiotherapy in the PSA era. Int J Radiat Oncol Biol Phys 2003;57:915-928.

3. Stephenson AJ, Eastham JA. Role of salvage radical prostatectomy for recurrent prostate cancer after radiation therapy. J Clin Oncol 2005;23:8198-8203.

4. Lerner SE, Blute ML, Zincke H. Critical evaluation of salvage surgery for radio-recurrent/resistant prostate cancer. I Urol 1995;154:1103-1109.
5. Rogers E, Ohori M, Kassabian VS, et al. Salvage radical prostatectomy: Outcome measured by serum prostate specific antigen levels. J Urol 1995;153:104-110.

6. Stephenson AJ, Scardino PT, Bianco FJ Jr, et al. Morbidity and functional outcomes of salvage radical prostatectomy for locally recurrent prostate cancer after radiation therapy. J Urol 2004;172:2239-2243.

7. Jamal K, Challacombe B, Elhage O, et al. Successful salvage robotic-assisted radical prostatectomy after external beam radiotherapy failure. Urology 2008;72:1356-1158.

8. Boris RS, Bhandari A, Krane LS, et al. Salvage roboticassisted radical prostatectomy: Initial results and early report of outcomes. BJU Int 2009;103:952-956.

9. Wei JT, Dunn RL, Litwin MS, et al. Development and validation of the expanded prostate cancer index composite (EPIC) for comprehensive assessment of health-related quality of life in men with prostate cancer. Urology 2000;56:899-905.

10. Rosen RC, Cappelleri JC, Smith MD, et al. Development and evaluation of an abridged, 5-item version of the International Index of Erectile Function (IIEF-5) as a diagnostic tool for erectile dysfunction. Int J Impot Res 1999;11:319-326.

11. Madi R, Daignault S, Wood DP. Extraperitoneal v intraperitoneal robotic prostatectomy: Analysis of operative outcomes. I Endourol 2007;21:1553-1157.

12. Pisters LL, Rewcastle JC, Donnelly BJ, et al. Salvage prostate cryoablation: Initial results from the cryo on-line data registry. J Urol 2008;180:559-564.

13. Ellis DS. Cryosurgery as primary treatment for localized prostate cancer: A community hospital experience. Urology 2002;60(suppl 1):34-39.

14. Han KR, Cohen JK, Miller RJ, et al. Treatment of organ confined prostate cancer with third generation cryosurgery: Preliminary multicenter experience. I Urol 2003;170:11261130.

15. Chin JL, Touma N, Pautler SE, et al. Serial histopathology results of salvage cryoablation for prostate cancer after radiation failure. J Urol 2003;170:1199-1202.

16. Huang WC, Kuroiwa K, Serio AM, et al. The anatomical and pathological characteristics of irradiated prostate cancers may influence the oncological efficacy of salvage ablative therapies. J Urol 2007;177:1324-1329.

17. Paparel P, Cronin AM, Savage C, et al. Oncologic outcome and patterns of recurrence after salvage radical prostatectomy. Eur Urol 2009;55:404-410.

Address correspondence to: Seth A. Strope, M.D., M.P.H.

Department of Urology

University of Michigan Health System 1500 East Medical Center Drive, TC 3875

Ann Arbor, MI 48109

E-mail: sethstro@umich.edu

\begin{aligned} & Abbreviations Used \\ & $\mathrm{CT}=$ computed tomography \\ & $\mathrm{EPIC}=$ Expanded Prostate Index Composite \\ & $\mathrm{PSA}=$ prostate-specific antigen \\ & $\mathrm{SHIM}=$ Sexual Health Inventory for Men \\ & \hline\end{aligned}


